\title{
RECHERCHES SUR LES CONDUITES POSSÉDANT DES RÉSERVOIRS D'AIR
}

Dans un mémoire récenl (1), M. Camichel, Professeur à la Facullé des Sciences el Directeur de l'Institut Electrolechnique de Toulouse publie les résultats de recherches non publiées jusqu'ulors sur les conduites possédant des réservoirs d'air.

La place nous manque pour donner comme il conviendrait ce travail en entier, mais nous en extrayons les applications praliques suivantes dont nos lecteurs pourront apprécier la valeur.

\section{Applicatrions piverses. \\ Remplissage des conduites.}

Dans les conditions de bassc chute, il se produit parfois, au moment du remplisságe, des oscillations en masse susceptibles de provoquer des pressions élevées; ces oscillations peuvent provenir du déplacement des grandes bulles existant dans la conduite.

D'une façon générale, il convient de faire arriver l'eau avec le maximum de lenteur compatible avec les conditions de l'installation et d'attendre que la purge de la conduite soit complète pour mottre en marche les turbines, la présence de bulles d'air étant susccptible de provoquer des phénomènes de résonance dangereux.

La vidange d'une poche d'air peut provoquer des pressions élevées ; l'cxpérience suivante le montre : on ouvre le robinet d'une poche d'air située à l'extrémité aval de la conduite de so millimètres (fig. 23) ; l'écoulement de l'air produit dans la

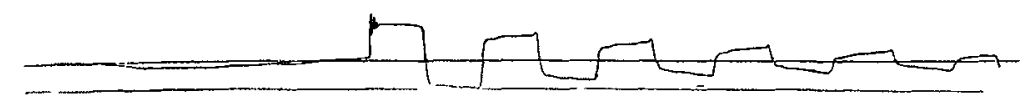

Fig. 23. - Surpression produite par la vidange d'une poche d'air; on voit que la surpression dépasse la pression statique; expé rience : $1 \mathrm{E}-162-6$.

conduite un coup de bélier d'ouverture qui s'amortit rapidement. La vitesse de l'cau dans la conduite a une vadeur sensiblement constante $v_{0}$. Au moment où l'air est complètement évacué, la vitesse de l'eau dans la conduite décroît brusquement jusqu'à une valeur très faible ; tout se passe comme dans le cas d'une fermeture de très courte durée. La surpression est très sensibllement égale à $\frac{a v_{0}}{g}$, elle peut dépasser beaucoup la pression statique.

Un accident de ce genre peut se produire quand un réservoir d'air branché sur une conduite en charge est fêlé par accident.

\section{Clapet automatique enregistreur de purge.}

On peut se rendre compte de l'état d'une conduite par l'examen des diagrammes de. fermeture, par la méthode de la dépression
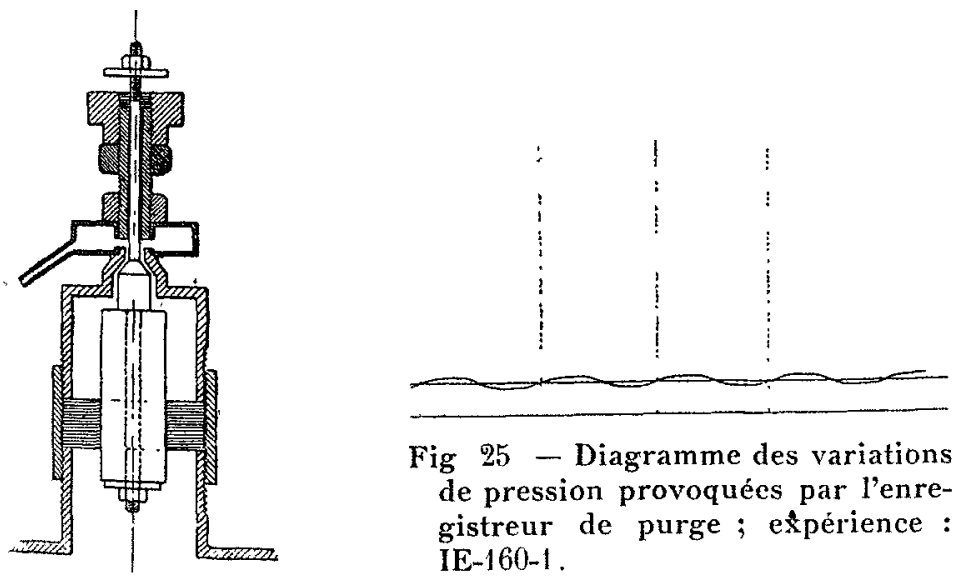

Fig 25 - Diagramme des variations de pression provoquées par l'enregistreur de purge; ex́périence : IE-160-1.

Fig. 24. - Clapet automatique enregistreur de purge.

brusque, etc., comme je l'ai indiqué, mais il est plus simple dans la pratique d'employer un clapet automatique (fig. 24) susceptible

(1) Publications de l'Ynstitut d'Electrotechnique et de Mécanıque appliquée de l'Université de Toulouse (1920). de donner, par son fonchonnement, que des varialions de pression très réduites; on y arrive facilement en munissant le clapet à sa partie inférieure d'une masse assez lourde pour qu'll s'ouvre dès que la pression devient légèrement inférleure à la pression statique. Un tachymètre actionné par le mouvement alternatif du clapet indique directement l'état de la conduite. La période de la conduite entièrement purgée est calculée a priori, ou bien détcrminée par l'expérience. La figure 25 indique le graphique des variations de pression indiquées par l'appareil.

\section{Emploi des ventouses pour la parge des conduiles.}

On emploie souvent pour éliminer les bulles d'air qui se pro. duisent dans les conduites des apparcils appelés ventouses. La figure 26 représente le schéma d'une ventouse de la maison d'Aubrives de Villcrupt. Le fonctionnement de ces appareils est très simple : quand une bulle entrainéc par lle mouvement do l'eau dans la conduite arrive en regard de la cloche A, l'air monte vers la partic supéricure de cette cloche et remplit la chambre H. Le clapet B tombe el démasque l'orifice C. L'air sous pression est évacué par la tubulure D. ce qui provoque un mouvement ascendant de l'eau dans la cloche $\mathrm{A}$ et une certaine vitesse $v_{0}$ dans la conduite.

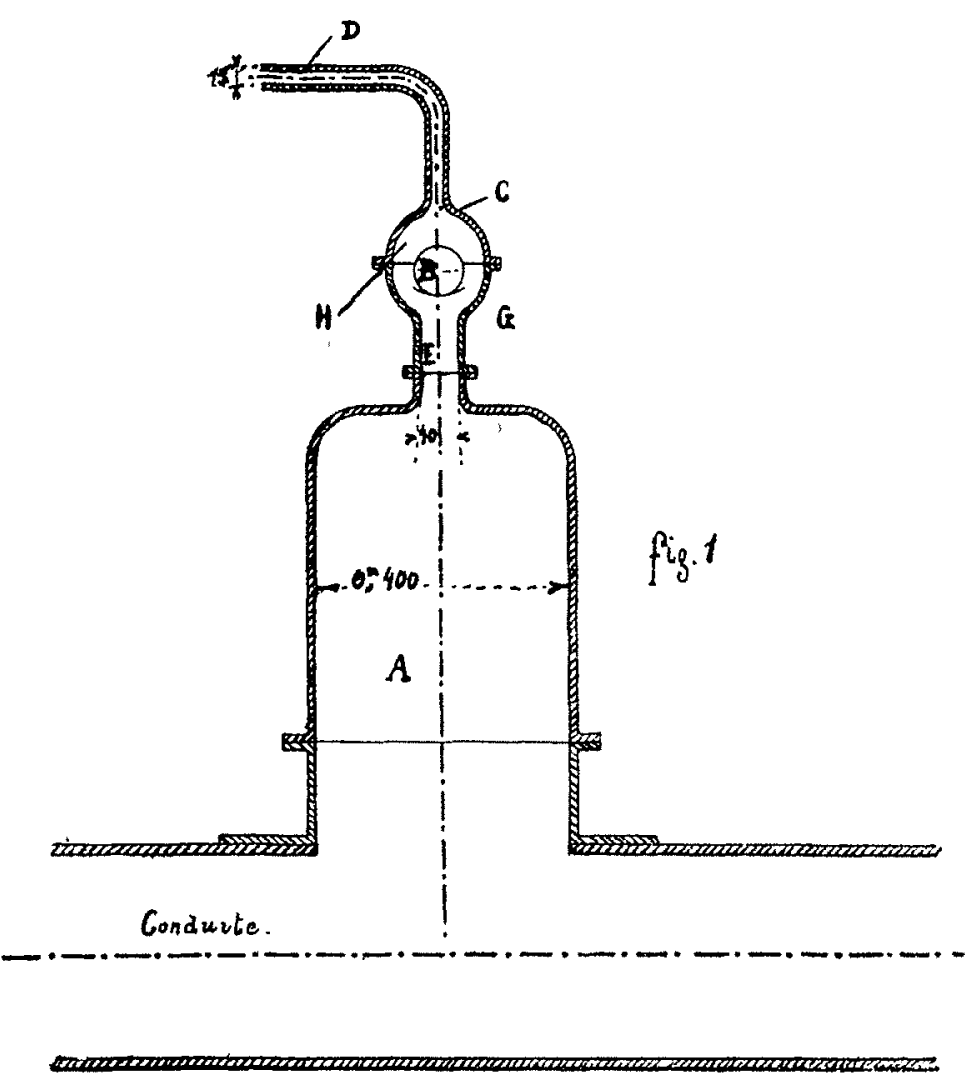

Fig. 26. - Ventouse.

Au moment où l'ear arrive au sommet de la cloche $\Lambda$, sa vitesse varie dans un intervalle de temps très court daus un rapport sensiblement égal au rapport des surfaces de la section do la cloche $\mathrm{A}$ et de la section de la tubulure $E$; pour les dimensions indiquées sur la figure 26 ce rapport est égal à $\frac{1}{100}$, les pertes de charge sont négligées. De pareilles variations de vitesse provoquent des surpressions élevécs susceptibles d'entraincr des ruptures de la conduite.

Dans une élude faite en collaboration avec M. l'ingénieur Espaignol, nous avons atténué très notablement ces surpreşsions, en ajoutant au bas de la trbulure $\mathrm{E}$ un tube percé de trous, comme le montre la figure 27 : la diminution de la vitesse de l'eau se produit d'une façon progressive, car les trous par lesqüels l'air 
peut sortir sont fermés à mesure que l'eau monte dans la cloche. La loi de rariation de la ritesse de l'eau dans la conduite peut être modifiéc à volonté en changcant le progresscur et la disposition des trous percés dans le tubc. Toul se passe donc comme si la conduile était munie d'un dishibuteur clont la vulesse de fermelare esl variable à volonlé.

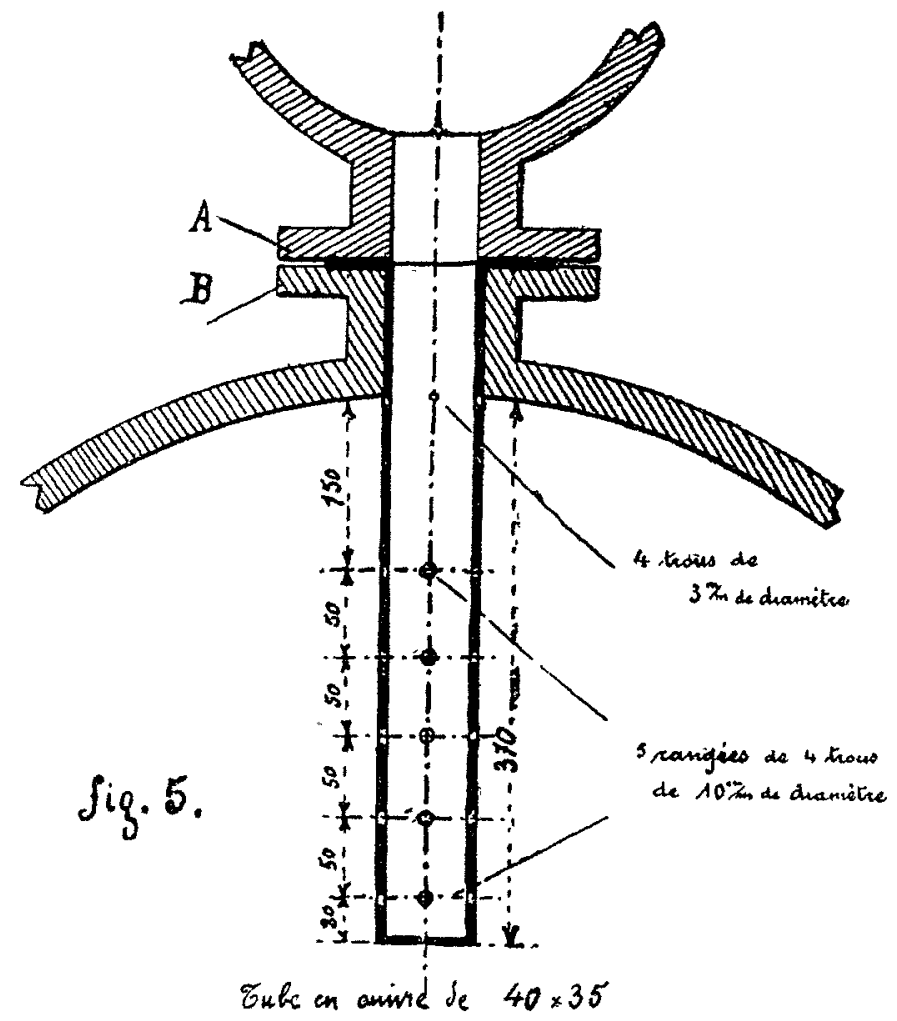

Fig. 27. - Ventouse munie d'un tube destinè à l'atténuation des coups de bélier.

Un autre procédé susceplible d'améliorer le fonctionnement des ventouses et d'éviter les ruptures de conduites consiste à subdiviser celles-ci en divers tronçons en les munissant, aux points les plus b́levés, de chambre d'eau ou de cheminées d'équilibre de grand diamètre. On réduit ainsi l'énergic accumulée dans la masse d'eau en mouvement au moment de la sortic de l'air $\left(^{1}\right)$.

A cet égard, on peut dire qu'une grosse bulle située dans une conduite est susceptible de protéger celle-ci en faisant l'office de chambre d'eau et produisant un véritable sectionnement de la conduite. A condition que le volume de cette bulle soit assez considérable, la prossion y.resta sensiblement constante et les réflexions s'y produisent avec changement de signe comme sur une chambre d'cau ; los surpressions n'attcignent pas les portions de la conduite situéos au-delà.

\section{Rem.irque sur l'enplor des motchteurs ; influence de tube} DE COMAUNICATION RELHANT TE MANOMÈTRE A LA CONDUTTE

En vue de l'emploi des indicateurs et du choix du tube mettant la conduite en communication avec celui-ci, on peut faire la remarque suivante $\left(^{2}\right)$ :

(1) Pour l'ótude des chemınces d'écullibre, consulter le Mémoire da M, Eydoux, Les mouvements de leau el les coups de bélier dans les cheminees d'equilibre (1919).

(1) Tous les systèmes élastiques sont ividemment susceptibles de provoquer des oscillations dans les conduites et, si leur importance est assez grande, des oscillations en masse. Dans les condurtes etudiées, J'ai ćvité d'employer des joints ćlästıques. Dans une conduite de 100 mètres de long à jounts de caoutchouc installée à l'usine à gaz de Toulouse, la fermelure du robinet située à l'extrémıté aval provocuart des déplacements de l'extrémité atteignant 25 contimètres d'amplitude. La conduite de 80 millimètres le l'Institut électrotechnique était formée de tronçons réunis entre eux par des raccords à vis sans interpositlon de joints de cagutchous.
Considérons un manomètre M. Jranché sur une conduile G par l'intermédiaire d'un tube de longucur $\mathrm{L}$ et de section $\mathrm{S}$. Le manomèlre, quel qu'il soit, indicateur, manomètres genre Bourdon, plaque métallique mince analogue ì celles employées dans les baromètres anéroides, enregistre les varialions de pression,

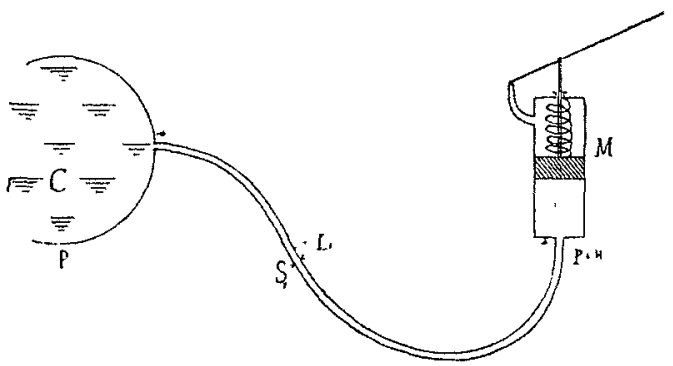

Fig. 28. - Schéma d'une conduite munie d'un indicateur.

par une déformation, qui provoque dans le lube de communi cation une mouvement en masse de l'cau. Soit $y+H$ la pression cn M, la pression au temps $l$ scra $\mathrm{H}(\mathrm{I}+z)+y, y$ la pression dans Ia conduite $\mathrm{C}$ au point où se trouve le tube do communication. Le théorème des forces vives donne, en désignant par $v$ la vilesse de l'eau dans le tube,

$$
\mathrm{HS}_{1} v d t+\left[y \mathrm{~S}_{1}-\left(y+\mathrm{H}(1+z) \mathrm{S}_{\mathrm{I}}\right\rfloor v d t=\frac{\mathrm{LS}_{1}}{g} v d v\right.
$$

ou

$$
\frac{\mathrm{L}}{g} \frac{d v}{d t}=-\mathrm{H} z
$$

La variation de pression $\mathrm{H} d z$ entraîne, par suite du déplacement du piston de l'indicateur, une variation de volume qui lui cst proportionnelle; en appelant $k$ le rapport de proportionnalité, on a :

$$
\mathrm{S}_{1} v d t=k \mathrm{H} d z
$$

d'où :

$$
\frac{\mathrm{L}}{g} \frac{k}{\mathrm{~S}_{1}} \mathrm{H} \frac{d^{2} z}{d t^{2}}=-\mathrm{H} z
$$

La période $\tau$ de l'oscillation en masse qui va prendre naissance sera :

$$
\tau=2 \pi \sqrt{\frac{\mathrm{L}}{g} \frac{k}{\mathrm{~S}_{1}}} \quad \text { posons }: \quad \mathrm{H} z=\mathrm{A} \sin \frac{2 \pi t}{\tau} .
$$

En désignant par $v_{0}$ la valeur de la vitesse $v$ ì l'époque zéro, où $\mathrm{H} z$ cst nul, on a :

$$
v_{0}=\frac{k}{S_{1}} A \frac{2 \pi}{\tau}
$$

dioù :

$$
\mathrm{A}=v_{0} \frac{\mathrm{S}_{1}}{k} \frac{\tau}{2 \pi}=v_{0} \frac{\mathrm{L}}{\mathrm{g}} \frac{2 \pi}{\tau}=v_{0} \sqrt{\frac{\mathrm{L}}{\mathrm{g}} \frac{\mathrm{S}_{1}}{k}}
$$

On pourra employer l'une ou l'autre de ces formules. Donnons un exemple:

Prenons un indicalcur à ressort exléricur (fig. 3 du lome 1) donnant une déviation du style de 60 millimètres pour une augmentation de pression de $x$, atmosphère; le piston de cet indicateúr a 2 centimètres de diamètre. Quand le style se déplace de 6 centimètres, le piston se déplace de I centimètre sculement. On a :

$$
\begin{gathered}
\pi \times 10^{-4} \times 10^{-2}=k \times 10, \\
k=\pi \times 10^{-7} .
\end{gathered}
$$


Voici une cxpérience IE- $33-9(f \circ g .29)$ :

$\mathrm{L}=15.3 ; d=11 \mathrm{~mm}$, diamètre du lube de communicalion

$H=1 / \mathrm{m} 7^{n}, v_{0}$ correspond à $\mathrm{mn}$ débil de $x 350 \mathrm{~cm}^{3}$ en $80^{5}$.

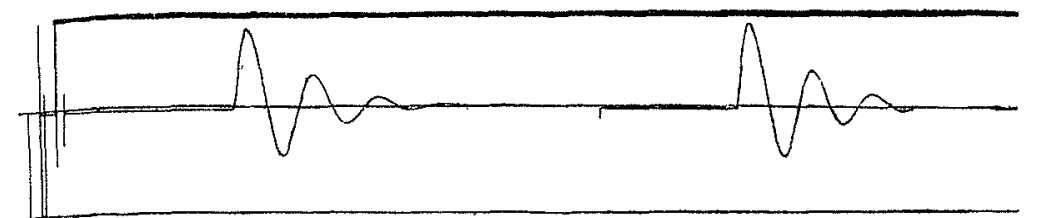

Fig. 29. - Influence du tube de communication placé entre le manomètre et la conduite; expérience : IE-138-8 et 9

Fous avons :

$$
\tau=2 \pi \sqrt{\frac{15,3 \times \pi \times 10^{-1}}{9,81 \times \pi \times \frac{1,1}{4} \times 10^{-4}}}=0^{s}, 45,
$$

on tronve $0^{\mathrm{a}}, 46$.

"u est donné par la formule:

$$
\begin{gathered}
v_{0} \times \pi \times \frac{\overline{1,1}^{2}}{4} \times 10^{-4}=\frac{1350}{80} \times 10^{-6}, \\
\text { d'où } v_{0}=0 \mathrm{~m} .18
\end{gathered}
$$

et

$$
1 \text { calc. }=\frac{1350}{80} \times \frac{4 \times 10^{-2}}{1,1^{2}} \times \frac{2 \times 15,3}{9,81 \times 0,45}=3 \mathrm{~m} .8 \pi \text {. }
$$

Comme sur le diagramme : $\operatorname{IE}_{-1} 38-9,27 \mathrm{~mm}$. ? correspondent it $/ \mathrm{m}$. $\mathrm{f}$ de pression, el que la surpression $\mathrm{A}$ est mesurée par y) 5 , on a :

$$
\text { A obs. }=\frac{4,7 \times 21,5}{27,2}=3 \mathrm{~m} .71 \text {; }
$$

on a exagérí, ì deasein, les perturbations dans les expériences prérédentes.

\section{Gamichel,}

Professem à la Faculte des Science-, Dirccleur de l'Institul electrotechnique de Toulouse.

\section{REVUE DES SOCIÉTÉS SAVANTES ET DES PUBLICATIONS SCIENTIFIQUES}

\section{ACADÉMIE DES SCIENCES}

\section{MÉCANIQUE APPLIQUÉE}

Sur une nouvelle forme canonique des massifs armés. - Sole de M. Charles Rabut.

Jusqu'à ce jour, les pièces métalliques mnies à du béton en ruc de créer un enscmble résistant ont affecté la forme linéaire, c'estàrlire celle de barres droites ou courbes tont la section peut avoir une forme quelconque, mais dont les dimensions transversales sont faibles par rapport à la dimension longitudinale. Dans une Note insérée aux Comples rendus (avril rgos), intitulée Lois de déformalion, principes de calcul el règles d'emploi scientifique du bélon armé, j’ai ćtabli que la formę canonique de cette combinaison comporle zéro, un, deux on trois conrs d'armatures tendues normales entre clles, dirigées en chaque point suivant les cfforts moléculaires principanx, sclon que, parmi ces efforts, il $y$ a soit zéro, soit une, soil deux, soil trois tensions, el n'exerçant sur le béton que des rézctions tangentielles suivant leur direction. Ces trois cours forment ainsi un systeme de lignes triplement or- thogonal dont la généralité n'est pas plus restreinte que celle du système des charges, cest-à-dire des forces extérieures auxquelles le massif doit résister.

Les nourclles conditions économiques créécs par la suerre, qui entrainent une révolution dans l'art de bàlir, m'ont conduit à cnvisagér, étudier et réaliser le remplaccment systémalique de l'armature-ligne par l'armature-surface, formée de plaques planes ou courbes dont l'épaisseur pcut, cn principe, varier suivant une loi quelconque, mais reste faible par rapport aux deux dimensions superficielles.

Getle combinaison comporte zéro, un ou deux cours d'armalures normales entre elles, orientées en chaque point parallelement à deux des trois efforts principaux, selon que parmi ces efforts il $y$ a soit zéro, soit une on deux, soit trois tensions, et n'excrçant sur le béton que des réactions tangenticlles; l'emploi de trois cours autour d'un point donné est toujours superflu puisque trois tensions trorthogonales peuvent se placer dans deux plans perpendiculaires, dont l'un posside nème un degré do liberté; il est toutefois avantageux que chacun de ces deux plans contienne deux des fensions principales, dont l'une soit la plus grande des trois ; cela posé, ot admeltant que les deux plus faibles tensions principales sont tangentes à un troisième cours virluel, on peut dre que l'armalure idéale complete forme $\mathrm{mm}$ système de surfaces triplement orthogonal, d'autant plus quo chacun des trois cours peut être réol dans certanes régions ot virtuel dans d'autres selon l'agencement des forces extórieures

Dans une région où les trois efforts principaux sont des tensions, on peut, au lien d'employer exclusivement des barrs ou des plaques, combiner un cours de plaques avec un cours de harres ; en chaque point, la barre est dirigée suivant une des tensions principales et la plaque est orientée suivant le plan des deux autres. Il faut remarquer d'ailleurs qu'un massif où tous les efforts intérieurs scraient des tensions n'a pas sa raison d'ètre, l'emploi du béton ne pourant être justifé que par l'existence et mème la prédominance des pressions. Le systìme mixte qui vient d'étre défini ne se réalisera done jamais scul el doit étre rationnellement considéré comme une transition motivée par l'emploi, dans deux parties contiguës d'une meme construetion, des deux formés canoniques simples, saroir : la forme acluelle (armature en barres) à laquelle restera attaché le nom d'llennebique, at la forme nouvelle (armature en plaques) qui est, à mon avis, celle de l'avenir.

Elle présente en eff'ct de nombreux arantages que l'évolution économique du monde tend à rendre de plus an plus grands, savoir :

Economie sur la main-d'œuvre nécessaire pour la mise en place et l'assujettissement d'un poids donné de métal ;

Plus grande stabilité avant et pendant l'enrobement;

Utilisation de la résistance dans deux directions principales d'efforts intérieurs au lieu d'une scule;

Pour une plaque enrobée sur ses deux côtés, indépendance des deux faces au point de vine de la dircction de l'cfiort tangenticl tolal, 'd'où augmentation du nombre des paramètres dont-on dispose pour serrer de plus près les données diun projet;

Pour une plaque cọrobée d'un seul còté, ohtention de la résistance maxima par l'éloignement maximum de l'axe neutre ; suppression de la tranche morte, c'est-ì-dire de la zone d'enrobement comprise entre l'armature et le parement, zone condamnéc à la fissuration et ne possérlant pas la même résistance que le reste du massif ;

Dans lo mème cas, protection du béton contre les chocs, les attaques chimiques, les infiltrations ;

Dans le mème cas encore, faculté d'uliliser l'armature comme coffrage ;

Dans tous les cas, possibilité d'augmenter "ou suppléer l'adhérence en soudant à la plaque des nervures métalliques transversales à l'effort tangentiel résultant el qui agissent par pression sur le béton ;

Enfin facilité de supprimer l'enrobement dans les régions où le métal est cxclusivement tendu. 